\title{
Adaptive morphogenesis of lamb liver under Anthropobiocenosis conditions lamb liver morphogenesis
}

\author{
T.P. Skobelskaya*, V.V. Lemeshchenko, N.V. Saenko, V.G. Sokolov, and E.V. Nekhaichuk \\ Federal State Autonomous Educational Institution of Higher Education "Crimean Federal University \\ named after V.I. Vernadskiy" of Agrotechnological Academy, 295492, Simferopol, Russia
}

\begin{abstract}
Investigated the histological features of the structure of hepar of one-day-old lambs (ODL) using a complex of morphological techniques. It was found that hepar in ODL is formed by stroma and parenchyma. The stromal elements of the organ are formed by loose lugs of hematopoietic connective tissue, which in turn forms a capsule (2.80-7.23 microns thick), as well as the septa of the lobules, which include the hepar triads and paravasal tissue of efferent blood vessels. As a result of the studies, the prevalence of the stroma in the hematopoietic foci around large afferent blood vessels, especially in the hepar hilus, was revealed. The hepar parenchyma in ODL has structural incompleteness. It is formed by hepar cells and numerous resinusoidal cells located in the subcapsular, peripheral, central zones in the lobus hepatis. Hepar cells located in the subcapsular zone (SZ) have a more optically cleared (OC) cytoplasm, and the practically cleared cytoplasm of the hepatocyte diameter (HD) decreases towards the central zone $(\mathrm{CZ})$. In the hepar parenchyma of the ODL, hematopoiesis foci (HF) are established in each of the lobes, which tend to decrease from the periphery to the center of the lobes. Hemopoetic cells (HC) are located between hepatocytes, densely layering on top of each other, and there are also cells located sparsely between the liver cells (LC). The number of hepatocytes per $1 \mathrm{~mm} 2$ of the area of the histological specimen in the left lobe (LL) of the ODL reaches the highest value $5983.79 \pm 90.40$, in the right lobe (RL) of the liver it is $5358.80 \pm 646.60$, while in the middle it has a minimum value and reaches $5133.10 \pm 205.75$.
\end{abstract}

\section{Introduction}

The productivity of an adult animal depends on its adaptive abilities at the newborn stage of development, manifesting itself in the implementation of the functions of all organs, including hepar $[4,6,9,10,11]$. Hepar performs a number of vital functions, and in particular, one of the main functions in the prenatal period is hematopoietic and the formation of the liver tissue itself. In the postnatal period, hepar undergoes a significant increase in functions against the background of attenuation of hematopoiesis, which is certainly reflected in the histological structure of hepar $[5,7,8]$. The lack of information

*Corresponding author: www.150171986@mail.ru 
about the structure of the hepar parenchyma and the degree of its incompleteness, as well as the performance of the main functions in newborn lambs, makes it difficult to clarify the features of pathomorphological changes in diseases of various etiologies.

There is practically no information in the literature on the structural incompleteness of hepar in lambs, which does not allow timely diagnosis and full prophylaxis of diseases in newborns, especially those occurring against the background of a general hematopoiesis inoculation of the body.

The aim of the study is to establish the hepar adaptive morphogenesis of male and female lamb under the conditions of an anthropically enlightened cytoplasmobiocenosis.

To achieve the goal, the following tasks were set:

- to establish the features of the structure of the parenchyma and stroma of the hepar in ODL;

- optically clarified cytoplasm to determine the peculiarities of intervascular and vascular-tissue relationships of hepar in male and female lambs.

\section{Materials and methods}

The morphological features of the parenchyma, stromal intervascular and vascular-tissue relationships of the hepar optically cleared cytoplasm were determined on histological organ preparations. For the preparation of histological sections, liver pieces with a thickness of $05-1 \mathrm{~cm}$ were taken, with an area of $1.5 \times 0.5 \mathrm{~cm}$ in the right, left hepar lobes perpendicular to the gate of the organ, the middle (caudate) lobe parallel to the hepar gate. The liver of the studied animals was fixed in a $10 \%$ formalin solution. The preparation of histological preparations with a thickness of 25-30 $\mu \mathrm{m}$ was carried out on a microtomecryostat MK-25-m at a temperature of $-12^{\circ} \mathrm{C} \ldots-16^{\circ} \mathrm{C}$. Sections of hematopoietic foci stained hematopoietic foci with silin and eosin [2]. In order to obtain thin (8-12 $\mu \mathrm{m})$ histological sections, the material was embedded in paraffin, which then stained the hematopoietic foci with Ehrlich's silin and eosin [3].

Morphometric parameters of hepar were determined using a Mikmed-5 binocular microscope. To establish the morphometric parameters of tissue components, the number of hepatocytes per $1 \mathrm{~mm}^{2}$ of the area of the hepar histological preparation, we used the Jeffreys method modified by S.A. Saltykov [1]. Longitudinal indicators of the structures were established using an eyepiece micrometer MOV-1-15 .

$$
X=z+0,5 w+0,25 u, \text { where }
$$

$\mathrm{X}$ - average number of hepatocytes in the cut area;

$\mathrm{z}$ - number of hepatocytes in the test system (ocular mesh insert);

$\mathrm{w}$ - number of hepatocytes crossed by the lines of the outer contour of the test system;

$\mathrm{u}$ - number of hepatocytes at the nodal points of the test system.

\section{Results}

In ODL, the tissue components of the hepar are represented by the stroma and parenchyma. The parenchyma is formed mainly by hepatocytes and a large number of resinusoid cells. In each lobes hepar of ODL, based on the morphological structure and its comparison with the stroma, it is possible to distribute: subcapsular (5.00-10.00 mm thick), peripheral (10.00$20.00 \mathrm{~mm}$ thick) and central (10.00- $28.00 \mathrm{~mm})$ zone. The organ stroma in JSV is formed by loose fibrous connective tissue forming a capsule (2.80-7.23 $\mu \mathrm{m}$ thick), septa of lobules, which have hepar triads and paravasal tissue of efferent blood vessels. Stromal elements 
prevail around the large afferent blood vessels, predominantly in the hepar hilum. Inside the hepar, the amount of stroma is reduced; it is formed only by thin collagen fibers around the hepatic triads and hepatic veins. In ODL, the stroma does not form the characteristic boundaries of the outline of the hepar lobules.

In the SZ of RL in the ODL, liver cells (LC) are predominantly rounded, less often oval HD of 12.13-16.10 $\mu \mathrm{m}$, the nuclei of the LC have a round or oval shape with HD from 3.97 $\mu \mathrm{m}$ to $7.23 \mu \mathrm{m}$. LC are close to each other, which creates a "honeycomb" look. But also insignificant areas were revealed in which LC form branched, short (3-7 hepatocytes) HD that anastomose with each other. The cytoplasm of hepatocytes $(\mathrm{CH})$ is about 68.00 $90.00 \%$ under the capsule in the RL in the of OC in the ODL, and about $10.00-32.00 \%$ is weakly eosinophilic, has a foamed appearance with different color intensity and different granularity (Fig. 1).

At the ODL LC in the SZ of RL, manifold branched beams are formed, the parameters of which are from $25.43 \times 35.23$ microns to $37.79 \times 41.53$ microns in diameter. Also distinctive is the formed single lobules (3-5 in the field of view of the microscope at a magnification of $10 \times 10$ ). $\mathrm{LC}$, which ranges from $257.59 \times 345.32$ microns to $444.25 \times 439.58$ microns. LC are formed by branched that anastomose with each other, as well as groups of cells that form a radial order relative to the central veins (CV) only in insignificant areas of the histological specimen (Fig. 2).

$\mathrm{HC}$ are located between hepatocytes compactly overlapping each other, and also cells located weakly sparse between the LC were revealed. These cells form islets that are round, oval or ovoid $11.90 \times 20.30 \mu \mathrm{m}$ to $13.76 \times 42.00 \mu \mathrm{m}$ in diameter. The islets are represented by densely scattered 3-29 small cells $2.10 \mu \mathrm{m}$ to $7.70 \mu \mathrm{m}$ and with a richly stained basophilic nucleus. The diameter of the sinusoidal capillaries (DSC) in the SZ is 6.07-7.23 microns.

In the peripheral zone (PZ) in RL the ODL, LC with a regular rounded shape prevail, and the HD is slightly smaller, in contrast to the SZ $(10.50-14.00 \mu \mathrm{m})$, while their nuclei are $4.67-6.30 \mu \mathrm{m}$. $\mathrm{CZ}$ is about $65.00-85.00 \%$ eosinophilic with various color saturation, and in $15.00-35.00 \%$ it is not completely OC, which is not inherent in such a ratio of cells under the hepar capsule.

In the PZ of the RL of the ODL, polymorphic branched beams are located, forming crowding with sizes from $22.16 \times 22.30 \mu \mathrm{m}$ to $76.52 \times 77.92 \mu \mathrm{m}$ in diameter, not ordered with respect to blood vessels. Thus, LC were identified that form anastomosing single beams, which have a radial orientation with respect to the $\mathrm{CV}$, which were detected only in certain areas of the histological specimen. It should be noted that the formed lobules reach $148.39 \times 129.73$ microns $-275.32 \times 301.46$ microns, and the PSC $-4.67-6.77$ microns. The hematopoietic islets (HI) in the PZ of the RL in the ODL have a polymorphic, oval or round shape. At the same time, LC are organized compactly or in a loose type. The number of cells in one islet in the PZ of the RL reaches 5-40, and that of the HD is 1.87-2.56 $\mu \mathrm{m}$ (Fig. $3,4)$. 


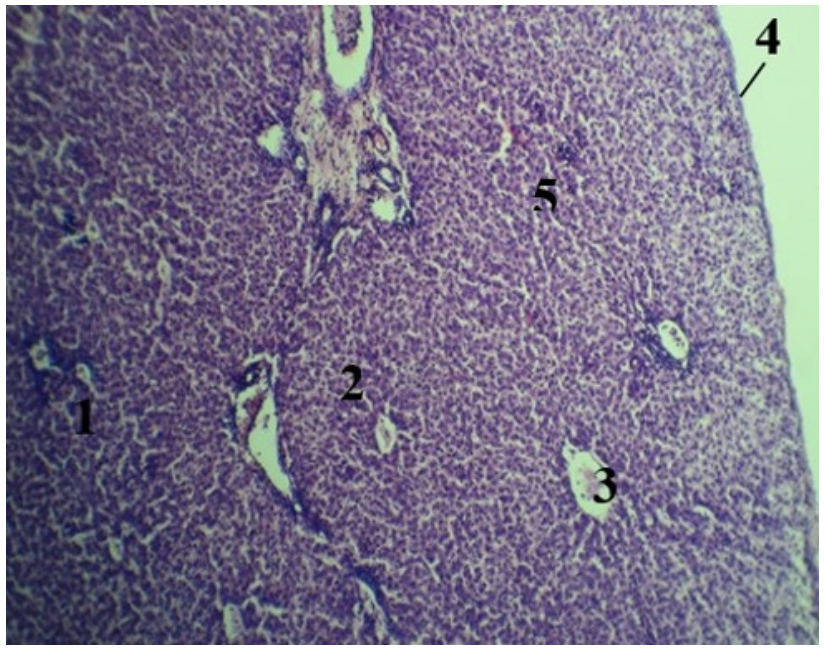

Fig. 1. Histological specimen of lamb liver. Age - day: 1 - hepatocytes; 2 - sinusoids, 3 - central vein; 4 - liver capsule; 5 - focus of hematopoiesis. Env. hematoxylin-eosin. Magnification - 100.

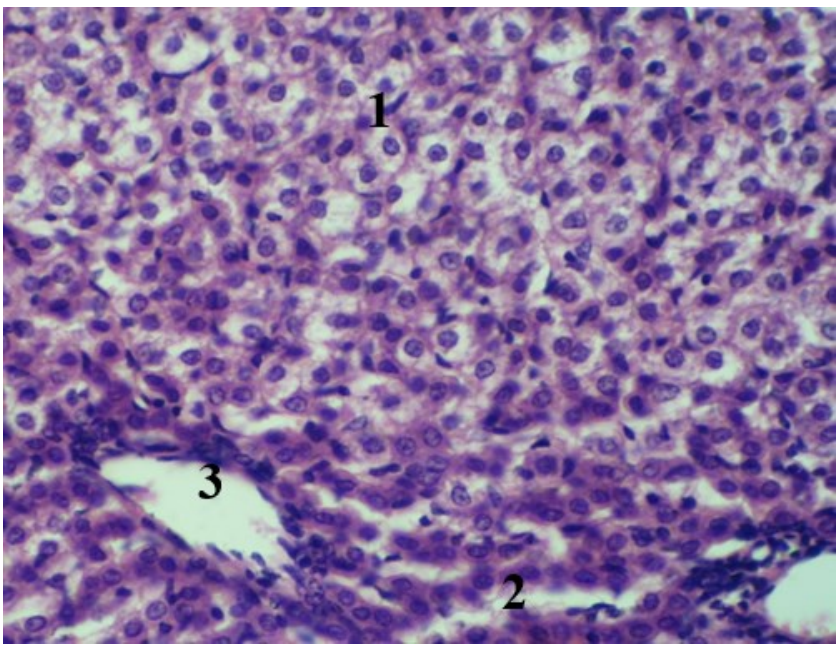

Fig. 2. Histological specimen of lamb liver. Age - day: 1 - hepatocytes; 2 - sinusoids, 3 - central vein. Env. hematoxylin-eosin. Magnification - 400.

The LC located in the central zone of the RL in the ODL have a rounded shape, and their diameter is 8.17-12.13 microns, while the nuclei (3.50-6.67 microns) are round. $\mathrm{CZ}$ of OC is about $10.00-25.00 \%$. At the same time, LV with OC by the cytoplasm is much higher than the cells (about 75-95\%), in which the cytoplasm is more stained. LC located near large II-III orders of blood vessels have a more strongly stained cytoplasm and a smaller diameter.

In the ODL LC, polymorphic branched clusters of beams are formed in the central zone of the RL, which are represented by an ovoid and oval shape $(42.46 \times 50.63-60.89 \times 70.22$ $\mu \mathrm{m})$. Single lobules formed by hepatocytes, which do not have a radial location in relation to blood vessels, with rare beams of hepatocytes with 5-9 cells and have a radial orientation in relation to $\mathrm{CV}$ only in certain areas of the histological specimen were revealed. The parameters of such lobules are $129.73 \times 215.59-195.06 \times 291.19 \mu \mathrm{m}$. DSC is 3.27-5.83 microns. HI in the central zone are small, oval and rounded, predominantly located compactly and reach $15.86 \times 19.83-23.80 \times 22.39 \mu \mathrm{m}$ in diameter. 
In the ODL, the number of hepatocytes located in the RL per $1 \mathrm{~m}^{\mathrm{m} 2}$ of the area of the histological specimen is $5358.80 \pm 646.60(\mathrm{~V}=12.07 \%)$.

In the SZ of the middle lobe of hepar (ML) in the ODL, the LC have a round, oval shape, and the HD is $13.06-15.63 \mu \mathrm{m}$, while the $\mathrm{CZ}$ is about $75.00-96.00 \%$ of the LC, which is characteristic of significantly large LC $4.00-25.00 \%$ weakly eosinophilic cytoplasm with variable granularity. The shape of the LC nuclei in the NW is round and has a diameter of 6.30-7.47 $\mu \mathrm{m}$.

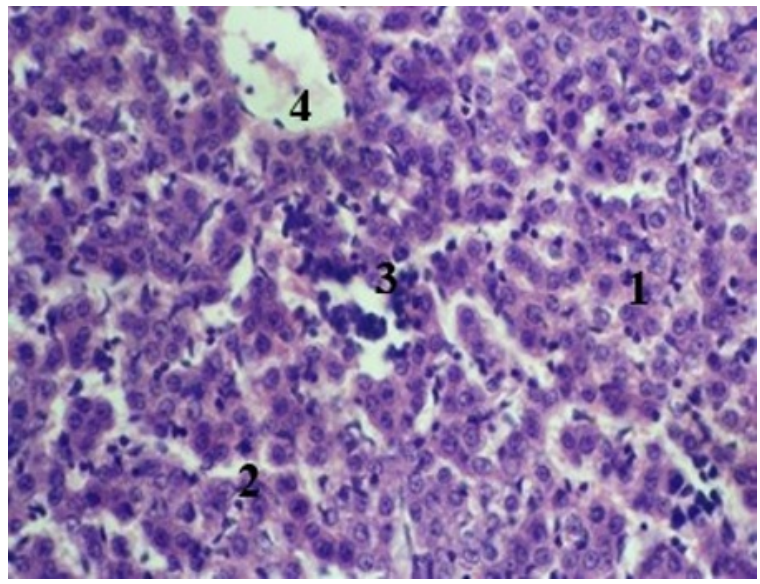

Fig. 3. Histological specimen of lamb liver. Age - day: 1 - hepatocytes; 2 - sinusoids, 3 - focus of hematopoiesis, 4 - central vein. Env. hematoxylin-eosin. Magnification - 400.

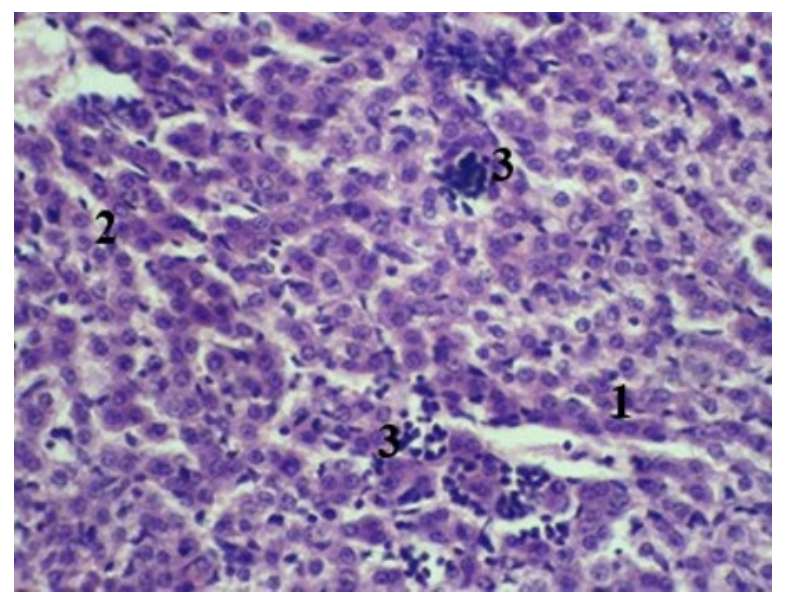

Fig. 4. Histological specimen of lamb liver. Age - day: 1 - hepatocytes; 2 - sinusoids, 3 - focus of hematopoiesis. Env. hematoxylin-eosin. Magnification - 400.

LC, which are located in the SZ of the ML at the ODL, are closely adjacent to each other, do not form an inherent radial beam structure. At the same time, they are combined into polymorphic groups of cells, the same pattern is also established in the RL, and their parameters reach from $15.16 \times 22.86 \mu \mathrm{m}$ to $26.13 \times 36.63 \mu \mathrm{m}$. The lobular structure of hepar in the SZ of the ML is much less pronounced, but single lobules that do not have definite boundaries were revealed, their parameters are $216.53 \times 169.86 \mu \mathrm{m}-293.06 \times 188.53 \mu \mathrm{m}$. The DSC in the SZ of the ML is 3.97-6.53 microns, while no HI were detected in this zone. 
The LC in the PZ of the ODL have a rounded shape with a diameter equal to 10.2611.67 microns, and their nuclei from 6.30 microns to 7.23 microns. $\mathrm{CZ}$ is about 55.00 $85.00 \%$ of OC, is $15.00-40.00 \%$ more strongly colored and $5.00-10.00 \%$ with slightly OD. In the PZ of the ML, the LC are not oriented in relation to the blood vessels and are organized into round, ovoid and oval multiforme groups of cells, and the diameter reaches from $30.79 \times 37.09$ microns to $47.36 \times 45.73$ microns. DSC in the PZ is $4.43-7.47$ microns. In ODL, only a small amount of hepar lobulation was revealed in the vicinity of large blood vessels. At the same time, the LC combined into beams are determined, the thickness of which reaches 9.80-13.76 microns, and also have a radial orientation to the efferent blood vessels. The parameters of these lobules are larger than those in the RL and reach from $296.79 \times 340.65$ microns to $376.12 \times 433.98$ microns.

In the ML of PZ in the ODL, LC have the shape of an oval, round or polymorphic shape, which contains small cells, densely packed or scattered with a strongly stained basophilic nucleus, the number of which in one islet varies from 4 to 48 ; their diameter is of 0.93-5.83 microns. HI parameters reach from $11.43 \times 26.83 \mu \mathrm{m}$ to $17.26 \times 29.63 \mu \mathrm{m}$.

The HD of the CZ ML in the ODL reaches 7.93-12.33 microns. Such LC are predominantly rounded, while their nuclei $(3.97 \mu \mathrm{m}$ to $5.83 \mu \mathrm{m})$ are rounded or oval, slightly basophilic. CZ is about $65.00-94.00 \%$ slightly eosinophilic, and the remaining LC are OC. CZ located near large blood vessels are much more stained, and in other areas LC with OC or slightly stained cytoplasm.

In the $\mathrm{CZ}$ of the ML in the ODL, LC are formed with branches that are anastomosed with each other, they have a radial placement in relation to the $\mathrm{CZ}$ only in insignificant areas of the histological specimen. Or they are defined in multiforme cell clusters from $28.70 \times 41.76 \mu \mathrm{m}$ to $49.69 \times 50.86 \mu \mathrm{m}$. Hepar lobules have indistinct boundaries and are equal from $328.52 \times 454.52$ microns to $405.99 \times 493.72$ microns, and DSC located in the central zone is $2.80-6.07$ microns.

The parameters of $\mathrm{HI}$ and $\mathrm{CZ}$ in the ML of the ODL range from $23.87 \times 34.53$ microns to $39.19 \times 60.42$ microns. The number of densely located LC in one focus is from 3 to 25 , and their parameters reach 3.50-5.37 microns, and the number of hepatocytes in the ML per 1 $\mathrm{mm}^{2}$ of the histological section area is slightly less than in the RL $-5133.10 \pm 205.75$ at $\mathrm{V}$ $=4.01 \%$.

LC located in the LL of the SZ in the ODL have an irregular rounded shape and a slightly smaller diameter of 8.87-10.27 $\mu \mathrm{m}$ than in the right and middle lobes of the organ. However, these cells are well isolated from each other, their cytoplasm is about 40.00$70.00 \%$ slightly eosinophilic, while $30.00-55.00 \%$ have OC. LC have rounded nuclei, their diameter is 4.90-6.07 microns. LC is formed by anastomosing beams going in different directions, creating a radial placement relative to the $\mathrm{CV}$ and only in insignificant areas of the histological specimen. In many areas, cells form multiforme clusters of a group of cells that are not radially located and reach from $28.00 \times 36.39 \mu \mathrm{m}$ to $44.56 \times 58.09 \mu \mathrm{m}$ in diameter.

It is necessary to point out that in the LL of the CZ in the ODL, the form of $\mathrm{HI}$ is oval or round, with a predominantly compact structure. OC are organized by LC with a rather strongly stained basophilic nucleus, their number in one focus can reach from 5-32 cells, while cell parameters vary at the border from 1.87-6.30 $\mu \mathrm{m}$. The HI index ranges from $22.86 \times 26.60$ microns to $30.56 \times 43.63$ microns in diameter. $\mathrm{HC}$ are located similarly to the right and middle lobes, that is, between hepatocytes.

The hepatocytes located in the LL of the PZ in the ODL are rounded with a diameter of 9.10-14.93 $\mu \mathrm{m}$, their nuclei are round or oval and reach 4.90-6.53 $\mu \mathrm{m}$. LC are compactly located to each other, and their cytoplasm is about $12.00-36.00 \%$ eosinophilic (with different color intensity), while about $68.00-88.00 \%$ of hepatocytes contain OC cytoplasm. 
It was also found that $\mathrm{LC}$ are not distributed oriented with respect to blood vessels, they are formed in polymorphic cell clusters (from $37.09 \times 49.23 \mu \mathrm{m}$ to $69.52 \times 72.32 \mu \mathrm{m}$ ).

In the LL of the PZ in the ODL, the formed lobules are detected only along the large blood vessels and range in diameter from $426.52 \times 485.31 \mu \mathrm{m}$ to $448.92 \times 526.38 \mu \mathrm{m}$. LC are connected in short (4-9 cells) branched beams, with a radial orientation in relation to the parenchymal venules. DSC reaches 3.03-6.77 microns.

In the LL of the PZ in the ODL, the forming lobules are detected only along the large blood vessels and range in diameter from $426.52 \times 485.31$ microns to $448.92 \times 526.38$ microns. LC are connected in short (4-9 cells) branched beams, with a radial orientation in relation to the parenchymal venules. DSC reaches 3.03-6.77 microns.

In the LL of the PZ in the ODL, HI are round, oval or ovoid and reach from $29.86 \times$ $28.69 \mu \mathrm{m}$ to $34.76 \times 48.76 \mu \mathrm{m}$ in diameter. $\mathrm{HC}$ are round, 0.93-5.37 microns in diameter, while their abundance in one focus can reach up to 42 cells.

In the $\mathrm{LL}$ of the $\mathrm{CZ}$ in the ODL, LC are round or oval with a diameter of 8.40-13.30 $\mu \mathrm{m}$, their nuclei are weakly basophilic, and their diameter is $3.50-6.77 \mu \mathrm{m}$. LC have eosinophilic cytoplasm with varying degrees of color (about 78.00-95.00\%), and about $5.00-25.00 \% \mathrm{HI}$ of the CZ. LC are formed into polymorphic groups of cells as in other parts of the hepar, but in diameter they range from $36.63 \times 45.73 \mu \mathrm{m}$ to $48.99 \times 56.23 \mu \mathrm{m}$.

The forming lobules, the sizes of which range from $235.19 \times 394.79 \mu \mathrm{m}$ to $352.79 \times 479.72 \mu \mathrm{m}$ in diameter, It should be noted that LC in the central zone of the LL are formed into branched beams, among which, in isolated cases, radially directed beams are revealed, reaching a length of 5-10 cells, which form a hepar lobule with fuzzy boundaries.

$\mathrm{HI}$ are formed by small $(1.87-4.67 \mu \mathrm{m})$ cells with an intensely stained nucleus, their number ranges from 5 to 39 cells in one focus. HI have not the same shape, but the most prevalent is oval, and the parameters of $\mathrm{HI}$ are from $23.80 \times 28.70 \mu \mathrm{m}$ to $34.76 \times 64.86 \mu \mathrm{m}$. The number of hepatocytes in the LL per $1 \mathrm{~mm}^{2}$ of the area of the histological section is the highest, reaching $5983.79 \pm 90.40$ with a small variability, reaching only $1.51 \%$.

\section{Conclusion}

Thus, in the parenchyma of the ODL, hepar has a structural incompleteness, which determines the metabolic activity of the organ. Located directly under the capsule in the SZ, especially the ML, the LC have predominantly OC cytoplasm, the beams in some areas are not differentiated. In the underlying PZ, the number of LC with cleared cytoplasm becomes less. They form branched beams, in rare cases uniting into forming lobules. The number of the latter is the greatest in the $\mathrm{CZ}$ of all hepar shares. In this case, the number of $\mathrm{HI}$ decreases from the periphery to the center of the lobes.

\section{References}

1. G. G. Avtandilov, Medical morphometry, 324 (1990)

2. N. P. Gavrilin, Actual nutrition of morphology, science practice of the II National Congress of Anatomists, Histologists, Embryologists and Topographers of Ukraine, 53 (1998)

3. A. P. Goralskiy, Fundamentals of histological technology and morphofunctional methods of preschooling in the norm with pathology, 288 (2005)

4. L. V. Kletikova, A. N. Martynov, N. P. Shishkina, D. I. Sinelshchikova, Bulletin of Agrarian Science, 1(82), 73 (2020)

5. O. A. Matveev, A. A. Torshkov, Bulletin of the Orenburg State Agrarian University, 2, 155 (2019) 
6. P. Praphatsorn, Asian Biomedicine, 4(4), 619 (2010)

7. S. M. Suleimanov, O. B. Pavlenko, V. S. Slobodyanik, L. P. Mironova, Izvestiya Orenburg State Agrarian University, 5, 182 (2019)

8. C. Stephens, Curr. Opin. Allergy Clin. Immunol, 14(4), 286 (2014)

9. P. S. Timkina, Agrarian science of Euro-North-East, 2(57), 51 (2017)

10. M. D. Tomoakilkeda, M. D. YujiMurata, J. Edward, M. D. Quilligan, T. Julian, M. D. Parer, American Journal of Obstetrics and Gynecology, 182(2), 449 (2000)

11. S. N. Chebakov, N. T. Silantyeva, Bulletin of the Altai State Agrarian University, 1(171), 110 (2019) 